\title{
Independent parental contributions initiate zygote polarization in Arabidopsis thaliana
}

\section{Authors}

Kai Wang ${ }^{1}$, Houming Chen ${ }^{1}$, Yingjing Miao ${ }^{1}$, Yanfei $\mathrm{Ma}^{2}$, Agnes Henschen ${ }^{1}$, Jan U. Lohmann ${ }^{2}$, Sascha Laubinger ${ }^{3}$, Martin Bayer ${ }^{1 *}$

\author{
Affiliations \\ ${ }^{1}$ Max Planck Institute for Developmental Biology, Department of Cell Biology, Max-Planck-Ring 5, \\ 72076 Tübingen, Germany \\ ${ }^{2}$ Department of Stem Cell Biology, Centre for Organismal Studies, Heidelberg University; 69120 \\ Heidelberg, Germany \\ ${ }^{3}$ University of Oldenburg, Institute for Biology and Environmental Sciences, Carl-von-Ossietzky- \\ Str. 9-11, 26111 Oldenburg, Germany \\ * correspondence: martin.bayer@tuebingen.mpg.de
}

\section{Keywords}

Arabidopsis thaliana, MAP kinase signaling, ERECTA signaling, embryogenesis, BRASSINOSTEROID SIGNALING KINASE, SHORT SUSPENSOR, cell polarity, zygote, suspensor, parental conflict

\section{Introductory paragraph}

Embryogenesis of flowering plants is initiated by polarization of the zygote, a prerequisite for correct axis formation in the embryo. In Arabidopsis, the zygote elongates about three-fold before it divides asymmetrically. The smaller apical daughter cell forms the pro-embryo while the larger basal cell is the precursor of the mostly extra-embryonic suspensor ${ }^{1}$. This filamentous organ plays a pivotal role in nutrient and hormone transport and is essential for the rapid growth of the embryo ${ }^{2}$. Zygote elongation and polarization is controlled by a MITOGEN-ACTIVATING PROTEIN (MAP) kinase signaling pathway including the MAPKK kinase YODA $\left(\mathrm{YDA} ;{ }^{3}\right)$. In yda mutants, the zygote divides symmetrically without obvious elongation. In many cases, the basal daughter cells follow an embryonic division pattern, yielding embryos with shorter or no suspensor. Constitutively active variants of YDA ( $y d a-C A$ ) give rise to a filament of daughter cells without recognizable proembryo ${ }^{3}$. Therefore, this pathway seems to control embryonic vs non-embryonic development in the early embryo.

Upstream of YDA act two members of the BRASINOSTEROID SIGNALING KINASE (BSK) family, $B S K 1$ and $B S K 2{ }^{4}$. These membrane-associated proteins serve as signaling relay linking receptor kinase complexes with intracellular signaling cascades ${ }^{5}$. In other developmental processes, YDA 
activity is controlled by receptor kinases of the ERECTA family (ERf), including ERECTA (ER), ERLIKE1 (ERL1), and ERL2 ${ }^{6-10}$. In the embryonic YDA pathway, however, receptor kinases upstream of BSK1 and BSK2 have so far not been identified ${ }^{1}$. Instead, YDA can in part be activated by the non-canonical BSK family member SHORT SUSPENSOR (SSP) by an unusual parent-of-origin effect ${ }^{4,11}$. SSP protein accumulates transiently in the zygote after fertilization presumably translated from sperm cell-derived transcripts. The SSP protein represents a naturally occurring, constitutively active variant of BSK1 and activates YDA as soon as it is present in the zygote ${ }^{4}$. SSP promotes suspensor formation and reinforces the stereotypic patterning of the early Arabidopsis embryo. Therefore, it has been speculated that SSP might be a paternal component of a parental tug-of-war controlling resource allocation towards the embryo ${ }^{2,11}$.

Here, we show that the receptor kinase ERECTA plays a crucial role in zygote polarization as maternally contributed part of the embryonic YDA pathway. SSP on the other hand provides an independent, paternal input to YDA activation, outlining a Y-shaped pathway. We conclude that two independent parental contributions initiate zygote polarization and control suspensor formation and embryonic development.

\section{Main}

The embryonic YDA pathway plays a central role in zygote polarization and the decision between embryonic versus non-embryonic development. It is however still unclear if this pathway is activated in response to extra-cellular signals. In many aspects of Arabidopsis development, YDA activity is controlled by receptor kinases of the ERECTA family ${ }^{7,8,10}$. As transcripts of $E R$ are present in the zygote (Table $\mathrm{S} 1 ;{ }^{12}$ ), a critical function of $E R$ in controlling zygote polarity seems plausible. However, homozygous er erl1 er/2 triple mutants are sterile and produce no embryos ${ }^{9}$. To address a possible function of $E R$ in early embryogenesis, we carefully examined homozygous single, double as well as segregating triple mutants of $E R f$ genes. When comparing er mutants with wildtype, weak defects in zygote elongation, zygote polarization and suspensor length became apparent (Figure 1 and Figure S1). Furthermore, aberrant division plane orientation in the suspensor can be observed (Figure S2). These defects are hallmarks of reduced YDA activity ${ }^{3}$ and mimic the phenotypes of ssp or bsk1 bsk 2 double mutants ${ }^{4,11}$. The erl1 erl2 double mutant did not show detectable differences to wildtype, indicating a minor role of $E R L 1$ and $E R L 2$ in early embryogenesis as long as a functional $E R$ is present. In the absence of $E R$, however, further loss of $E R L 1$ or $E R L 2$ enhanced the mutant phenotype, suggesting that they can partially take over $E R$ function (Figure 1 and Figure S1). This is reminiscent of ERf function during stomata development, where individual ERf members have by and large distinct signaling functions but can take over the role of others in their absence ${ }^{8,13-15}$.

To test if ER acts upstream of YDA in the context of zygote polarization as it does in other developmental contexts ${ }^{8,15,16}$, we performed genetic rescue experiments with a constitutively active version of YDA ( $y d a-C A^{3}$; Figure S3). Constitutive YDA activity rescued the er er/2 double mutant phenotype and resulted in a similar gain-of-function phenotype as observed in $y d a-C A$, suggesting a function of $E R$ and $Y D A$ in a common signaling cascade. Triple $E R f$ mutants with segregating er (er+/-erl1 er/2), however, did not show defects in zygote elongation or suspensor formation despite normal transmission of the er mutant allele (Figure1, Table S2). The fact that phenotypic defects in homozygous embryos only became apparent if the parental plant was homozygous, indicated possible parent-of-origin effects. We tested this by analyzing F1 embryos of reciprocal crosses of er erl double mutants and wildtype (Figure 2 and Fig. S4). In these 
crosses, we could only observe embryonic defects if the maternal plant carried the er erl mutations but not if these alleles were transmitted by the pollen donor, indicating that ER function is under maternal control (Fig. 2, and Fig. S4). Furthermore, zygote elongation defects could not be observed if the maternal plant was heterozygous for the er mutation (Fig. 1), although slightly altered zygote polarity can be observed in the segregating triple er $+/$ - er/1 er/2 mutant (Fig. S1). The phenotype of the embryo therefore seems to be predominantly determined by the genotype of the maternal sporophyte while loss of $E R$ function in the haploid phase or the embryo in segregating plants did not seem to have a major effect on the embryonic phenotype. This unusual parental effect may explain why a role of ERECTA in zygote polarization has so far been overlooked.

Two possible scenarios could explain the sporophytic maternal effect: Zygote polarization could be affected by a non-cell autonomous function of ER in sporophytic tissue. Such a scenario has for example been described for early auxin signaling in the embryo ${ }^{17}$. Alternatively, ER could function in the zygote but relying on $E R$ transcripts and/or ER proteins inherited to the zygote via the pre-meiotic megaspore mother cell (MMC).

A transcriptional ( $p E R:: 3 x$ Venus-N7) and a functional translational fusion ( $p E R:: E R-Y P e t$ ) reporter gene revealed $E R$ expression in the sporophytic seed coat as well as the female germ line (Fig. S5). As ER transcripts can be detected in isolated egg cells and zygotes (Table S1; ${ }^{12}$ ), we were not able to draw a definite conclusion about the location of ER function based on the observed expression pattern. However, $y d a$ is a zygotic recessive mutant ${ }^{3}$ (Figure 1, Figure S1) and SSP acts as sperm-derived activator, arguing for a function of the embryonic ERECTA/YDA pathway in the zygote. When looking at mRNA stability, $E R$ transcripts showed a moderate half-live in seedlings at roughly the same level as typical house-keeping genes, such as ACTIN 2, for which rapid $m R N A$ turn-over does not appear necessary (Table S3) ${ }^{18}$. ER transcripts can be detected in the egg cell and early zygote but transcript levels decline after fertilization, indicating that there is little or no de novo transcription in the zygote ${ }^{12}$ (Table S1). In order to address the question where ER function is necessary for zygote polarization, we specifically reduced ER protein function in the zygote using the $p E R:: E R$-YPet line in er erl1 erl2 background. Genetically encoded anti-GFP nanobodies fused to ubiquitin ligases have been used as elegant tool to specifically target GFP/YFP-tagged proteins for degradation ${ }^{19,20}$. To first test its functionality, we expressed the NSImb-vhhGFP4 nanobody under the $E R$ promoter. The resulting seedlings mimicked the er erl1 erl2 triple mutant phenotype albeit with a slightly weaker phenotype (Fig. 3), indicating that the nanobody is functional but does not completely abolish ER-YPet activity. In a next step, we expressed the nanobody under the strong egg cell-specific EC1 promoter to specifically reduce ER-YPet levels in the egg cell/zygote. This resulted in a significant reduction of zygote length in the $p E R:: E R$-YPet rescue line, indicating that zygote polarization critically depends on functional $E R$ protein in the zygote. Taken together, the available data suggests that the maternal control of ER function can be explained by the inheritance of pre-meiotically produced $E R$ transcripts to the zygote, compensating the loss of ERECTA in the female germline.

BSK1 and BSK2 have been shown to act upstream of YDA, presumably linking ERECTA signaling with YDA activation ${ }^{4}$. Reciprocal crosses with bsk1 bsk2 double mutants and wildtype showed similar sporophytic maternal effects as observed for er (Figure S7). In addition, maternal effects have been described for mutations in genes acting downstream of YDA ${ }^{21,22}$. Therefore, not just $E R$ but several components of this signaling pathway appear to be under maternal control. This is 
quite intriguing, as the noncanonical BSK family member SSP activates YDA as paternal component contributed by the sperm cell ${ }^{11}$. SSP has been shown to resemble a naturally occurring, constitutively active form of BSK that directly interacts with YDA via its TPR motif ${ }^{4}$. This raises the question whether the two parental contributions to YDA activation can function independently. We tested this hypothesis in seedlings, where ectopic expression of SSP leads to a strong gain-of-function phenotype by constitutive activation of the YDA pathway ${ }^{4,11}$. Ectopic expression of SSP-YFP ( $p 35 S:: S S P-Y F P$ ) in wildtype resulted in seedlings with cotyledons lacking mature stomata (Figure 4). This was also observed for SSP-YFP expression in er erl1 erl2 triple mutant background, indicating that SSP can activate YDA in the absence of a functional receptor complex.

If SSP indeed serves as independent signal for YDA activation in the zygote, loss of SSP should have an additive effect to loss of $E R$ function. We therefore observed the zygotic phenotype of ssp, er erl, and er erl ssp mutants (Figure 2 and Fig. S8). The further loss of SSP in er erl1 mutant lead to further reduction in zygote length and an almost complete loss of zygote polarity. Taken together, this indicates that SSP provides additional YDA activation that is independent of $E R$ function. If the embryonic YDA pathway indeed relies on two independent signaling inputs, additional SSP activity should compensate for the loss of ER function. To test this, we introduced an additional copy of SSP ( $P S S P:: S S P-Y F P$ ) in the er er/2 double mutant and found that additional $S S P$ activity indeed partially rescued the loss-of-function phenotype of er erl2 (Figure 4).

Taken together, our data outlines a Y-shaped signaling pathway (Figure 4e) with a maternally controlled receptor complex and a paternally provided activating protein that converge at the level of MAP3K activation. SSP is a Brassicaceae-specific gene while ERECTA, BSK1, and BSK2 are evolutionarily conserved in flowering plants ${ }^{23,24}$. SSP has been shown to be critical for correct suspensor development, an organ that serves as conduit for nutrients to the embryo, and for rapid development of the embryo ${ }^{2}$. Therefore, suspensor development could be a possible target of a parental conflict over nutrient allocation to the embryo. It has recently been observed that there are preferentially maternal expressed genes in the suspensor while there is mainly biparental expression in the apical cell ${ }^{25}$. This maternal bias in the suspensor could be interpreted in the context of the parental conflict theory enforcing equal nutrient distribution to all embryos by the maternal genome ${ }^{26}$. In this scenario, SSP could be seen as a Brassicaceaespecific, paternal contribution that circumvents the maternal control over suspensor development by bypassing ERECTA signaling as strong activator of the YDA pathway.

This raises the question if suspensor development in other plant species similarly is also under maternal control and if other parental contributions exist. It will be fascinating to elucidate whether the two independent inputs for YDA activation indeed are part of a parental tug-of-war over nutrient allocation to the embryo or if Brassicaceae are shifting from ligand-/receptor-based zygote polarization to a sperm-based activation mechanism via SSP to fine-tune the timing of YDA activation as SSP naturally links fertilization with YDA activation.

\section{Acknowledgements}

We would like to thank Gerd Jürgens for helpful discussions and comments on the manuscript. We also thank the Salk Institute Genomic Analysis Laboratory as well as the Nottingham Arabidopsis Stock Centre (NASC) for providing the sequence-indexed Arabidopsis T-DNA insertion mutants. Research in our groups is supported by the German Research Foundation (Deutsche 
Forschungsgemeinschaft - DFG SFB1101/B01 to M.B. and SFB1101/B07 to JUL) and the Max Planck Society.

\section{Author contributions}

KW and MB designed the study with critical comments and modifications by HC, YMi, YMa, JUL, and SL. KW, HC, YMi, and $A H$ performed the experiments. KW, $\mathrm{HC}, \mathrm{YMi}, \mathrm{YMa}, \mathrm{AH}, \mathrm{JUL}, \mathrm{SL}$, and $\mathrm{MB}$ evaluated the data and interpreted the experimental results. $\mathrm{KW}$ and $\mathrm{MB}$ wrote the initial manuscript draft, all authors commented on and edited the manuscript.

\section{Competing interests}

The authors declare no competing interests.

\section{References:}

1 Wang, K., Chen, H., Miao, Y. \& Bayer, M. Square one: zygote polarity and early embryogenesis in flowering plants. Curr Opin Plant Biol, doi:10.1016/j.pbi.2019.10.002 (2019).

2 Babu, Y., Musielak, T., Henschen, A. \& Bayer, M. Suspensor Length Determines Developmental Progression of the Embryo in Arabidopsis. Plant Physiology 162, 1448-1458, doi:DOI 10.1104/pp.113.217166 (2013).

3 Lukowitz, W., Roeder, A., Parmenter, D. \& Somerville, C. A MAPKK kinase gene regulates extra-embryonic cell fate in Arabidopsis. Cell 116, 109-119, doi:S0092867403010675 [pii] (2004).

4 Neu, A. et al. Constitutive signaling activity of a receptor-associated protein links fertilization with embryonic patterning in Arabidopsis thaliana. Proc Natl Acad Sci U S A 116, 5795-5804, doi:10.1073/pnas.1815866116 (2019).

5 Kim, T. W. \& Wang, Z. Y. Brassinosteroid signal transduction from receptor kinases to transcription factors. Annu Rev Plant Biol 61, 681-704, doi:10.1146/annurev.arplant.043008.092057 (2010).

6 Torii, K. U. et al. The Arabidopsis ERECTA gene encodes a putative receptor protein kinase with extracellular leucine-rich repeats. Plant Cell 8, 735-746, doi:10.1105/tpc.8.4.735 (1996). Bergmann, D. C., Lukowitz, W. \& Somerville, C. R. Stomatal development and pattern controlled by a MAPKK kinase. Science 304, 1494-1497, doi:10.1126/science.1096014 304/5676/1494 [pii] (2004). differentiation by synergistic interactions of receptor kinases. Science 309, 290-293, doi:309/5732/290 [pii] 10.1126/science.1109710 (2005).

9 Shpak, E. D., Berthiaume, C. T., Hill, E. J. \& Torii, K. U. Synergistic interaction of three ERECTAfamily receptor-like kinases controls Arabidopsis organ growth and flower development by promoting cell proliferation. Development 131, 1491-1501, doi:10.1242/dev.01028 (2004).

10 Meng, X. et al. A MAPK cascade downstream of ERECTA receptor-like protein kinase regulates Arabidopsis inflorescence architecture by promoting localized cell proliferation. Plant Cell 24, 4948-4960, doi:10.1105/tpc.112.104695 (2012).

11 Bayer, M. et al. Paternal control of embryonic patterning in Arabidopsis thaliana. Science 323, 1485-1488, doi:323/5920/1485 [pii] 10.1126/science.1167784 (2009). 
$13 \mathrm{Qi}$, X. et al. Autocrine regulation of stomatal differentiation potential by EPF1 and ERECTALIKE1 ligand-receptor signaling. eLife 6, doi:10.7554/eLife.24102 (2017).

14 Ho, C. M., Paciorek, T., Abrash, E. \& Bergmann, D. C. Modulators of Stomatal Lineage Signal Transduction Alter Membrane Contact Sites and Reveal Specialization among ERECTA Kinases. Dev Cell 38, 345-357, doi:10.1016/j.devcel.2016.07.016 (2016).

15 Shpak, E. D. Diverse roles of ERECTA family genes in plant development. J Integr Plant Biol 55, 1238-1250, doi:10.1111/jipb.12108 (2013).

16 Hara, K., Kajita, R., Torii, K. U., Bergmann, D. C. \& Kakimoto, T. The secretory peptide gene EPF1 enforces the stomatal one-cell-spacing rule. Genes Dev 21, 1720-1725, doi:10.1101/gad.1550707 (2007).

17 Robert, H. S. et al. Maternal auxin supply contributes to early embryo patterning in Arabidopsis. Nature plants 4, 548-553, doi:10.1038/s41477-018-0204-z (2018).

18 Szabo, E. X. et al. Metabolic Labeling of RNAs Uncovers Hidden Features and Dynamics of the Arabidopsis Transcriptome. Plant Cell 32, 871-887, doi:10.1105/tpc.19.00214 (2020).

19 Caussinus, E., Kanca, O. \& Affolter, M. Fluorescent fusion protein knockout mediated by antiGFP nanobody. Nat Struct Mol Biol 19, 117-121, doi:10.1038/nsmb.2180 (2011).

20 Ma, Y. et al. WUSCHEL acts as an auxin response rheostat to maintain apical stem cells in Arabidopsis. Nat Commun 10, 5093, doi:10.1038/s41467-019-13074-9 (2019).

21 Zhang, M. et al. Maternal control of embryogenesis by MPK6 and its upstream MKK4/MKK5 in Arabidopsis. Plant J 92, 1005-1019, doi:10.1111/tpj.13737 (2017).

22 Ueda, M. et al. Transcriptional integration of paternal and maternal factors in the Arabidopsis zygote. Genes Dev 31, 617-627, doi:10.1101/gad.292409.116 (2017).

23 Liu, S. L. \& Adams, K. L. Dramatic Change in Function and Expression Pattern of a Gene Duplicated by Polyploidy Created a Paternal Effect Gene in the Brassicaceae. Mol Biol Evol, doi:msq169 [pii] 10.1093/molbev/msq169 (2010).

24 Caine, R. S. et al. An ancestral stomatal patterning module revealed in the non-vascular land plant Physcomitrella patens. Development 143, 3306-3314, doi:10.1242/dev.135038 (2016).

25 Zhao, P., Zhou, X., Zheng, Y., Ren, Y. \& Sun, M. X. Equal parental contribution to the transcriptome is not equal control of embryogenesis. Nature plants 6, 1354-1364, doi:10.1038/s41477-020-00793-x (2020).

26 Haig, D. Kin conflict in seed plants. Trends Ecol Evol 2, 337-340, doi:0169-5347(87)90110-8 [pii] 10.1016/0169-5347(87)90110-8 (1987).

\section{Figure legends}

Figure 1: Zygote polarity and suspensor development

a-h) DIC images of cleared ovules showing representative 1-cell embryos. Apical cells are false colored in yellow, basal cells in blue. The genotype is given below the image. Heterozygous parental lines are indicated by $+/-$. Scale bar $=10 \mu \mathrm{m}$.

i) Box plot diagram of zygote length in $\mu \mathrm{m}$. The sample size is given above $\mathrm{x}$-axis. Center lines show the medians; box limits indicate the 25 th and $75^{\text {th }}$ percentiles; whiskers extend 1.5 times the interquartile range from the 25 th and 75 th percentiles; crosses represent sample means; data points are plotted as grey dots. Statistical differences determined by Mann-Whitney U-test $(p<0.05)$ are indicated by different letters above graph. In embryos of heterozygous yda plants, two distinct populations of data points can be observed, with approximately a quarter showing strong zygote elongation defects possibly representing the homozygous offspring.

$\mathrm{j}$-q) DIC images of cleared ovules showing transition stage embryos. The genotype is given below the image. Heterozygous parental lines are indicated by $+/$-. Scale bar $=20 \mu \mathrm{m}$. 
r) Box plot diagram of suspensor length at transition stage. The sample size is given above the $x$-axis. Center lines show the medians; box limits indicate the 25th and 75th percentiles; whiskers extend 1.5 times the interquartile range from the 25 th and 75 th percentiles; crosses represent sample means; data points are plotted as grey dots. Statistical differences determined by Mann-Whitney U-test $(p<0.05)$ are indicated by different letters above graph. In embryos of heterozygous yda plants, two distinct populations of data points can be observed, with approx. a quarter showing reduced suspensor length possibly representing the homozygous offspring.

\section{Figure 2: Parental effects in reciprocal crosses}

Box plot diagrams of zygote elongation a) and suspensor length b) in $\mu \mathrm{m}$ in F1 embryos of reciprocal crosses. The embryonic defects of the er erl 2 double mutant depends solely on the maternal genotype. The paternal effect of ssp mutants enhances the er er/2 defects in er erl 2 x ssp. The reciprocal ssp x er er/2 cross does not show any detectable differences to wildtype. Genotypes are given as female $x$ male. The sample size is indicated above the $x$-axis. Center lines show the medians; box limits indicate the 25th and 75th percentiles; whiskers extend 1.5 times the interquartile range from the 25th and 75th percentiles; small crosses represent sample means; data points are plotted as grey dots. Statistical differences determined by Mann-Whitney U-test $(p<0.05)$ are indicated by different letters above graph.

\section{Figure 3: Cell-autonomous function of ER protein in the zygote}

Vegetative growth phenotype of wild-type Col-0 (a), er erl1 erl2 triple homozygous (b), genetically rescued plants ( $p E R:: E R$-YPet in er erl1 erl2; $c$ ), and genetically recued plants expressing anti-GFP nanobody ( $p E R:: N S I m b$-vhhGFP4 in pER::ER-YPeter erl1 erl2; d). Scale bar $=1 \mathrm{~cm}$.

e) Egg cell-specific expression of the anti-GFP nanobody ( $p E C 1:: N S I m b$-vhhGFP4) in the $p E R:: E R-Y P e t$ er erl1 er/2 plant leads to reduced zygote length. Box plot diagram of zygote elongation in $\mu \mathrm{m}$. The sample size is indicated above the $x$-axis. Center lines show the medians; box limits indicate the 25th and 75th percentiles; whiskers extend 1.5 times the interquartile range from the 25th and 75th percentiles; small crosses represent sample means; data points are plotted as grey dots. Statistical differences determined by Mann-Whitney $\mathrm{U}$-test $(p<0.05)$ are indicated by different letters above graph.

Figure 4: Y-shaped topology of the embryonic YDA pathway

Ectopic expression of SSP-YFP in leaves under control of the CaMV 355 promoter ( $p 355::$ SSP-YFP) leads to absence of stomata on the leaf surface in wild-type (c) and er erl1 erl 2 background (d). Col-0 (a) and er erl1 er/2 (b) are shown as reference. SSP-YFP signal is shown in green, propidium iodide staining in red. Scale bar $=20 \mu \mathrm{m}$.

An additional copy of SSP ( $P S S P:: S S P$-YFP) partially rescues the er erl 2 loss-of-function phenotype in the zygote (e). Box plot diagram of zygote elongation in $\mu \mathrm{m}$. The sample size is indicated above the $\mathrm{x}-$ axis. Center lines show the medians; box limits indicate the 25th and 75th percentiles; whiskers extend 1.5 times the interquartile range from the 25th and 75th percentiles; small crosses represent sample means; data points are plotted as grey dots. Statistical differences determined by MannWhitney $\mathrm{U}$-test $(\mathrm{p}<0.05)$ are indicated by different letters above graph. 
f) Simplified schematic depiction of the embryonic YDA pathway. YDA is activated by the maternally supplied receptor complex consisting of ERECTA and the membrane-associated proteins BSK1 and BSK2. YDA can also be activated by the paternally contributed BSK family member SSP. Signaling components are colored according to genetic contribution: maternal effect (green), paternal effect (red), zygotic recessive (grey).

\section{Supplementary data:}

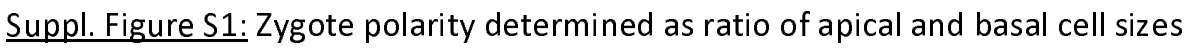

Suppl. Figure S2: Frequency of aberrant division planes in the suspensor

Suppl. Figure S3: Genetic rescue with yda-CA

Suppl. Figure S4: Sporophytic maternal effect of er erl1 double mutant

Suppl. Figure S5: ERECTA expression in developing ovules

\section{Suppl. Figure S6: Expression of $p E R:: E R-Y P e t$ in er erl1 erl2}

Suppl. Figure S7: Sporophytic maternal effect of bsk1 bsk2 double mutants

Suppl. Figure S8: Parental effects of er erl1 and ssp mutations.

Supplementary Table S1: mRNA abundance of ERECTA family genes during early embryogenesis.

Supplementary Table S2: Segregation rates of $E R$ and $E R L 1$ in triple mutant combinations

Supplementary Table S3: mRNA decay of selected candidate genes

Supplementary Table S4: primers and oligo nucleotide sequences

Material and Methods 

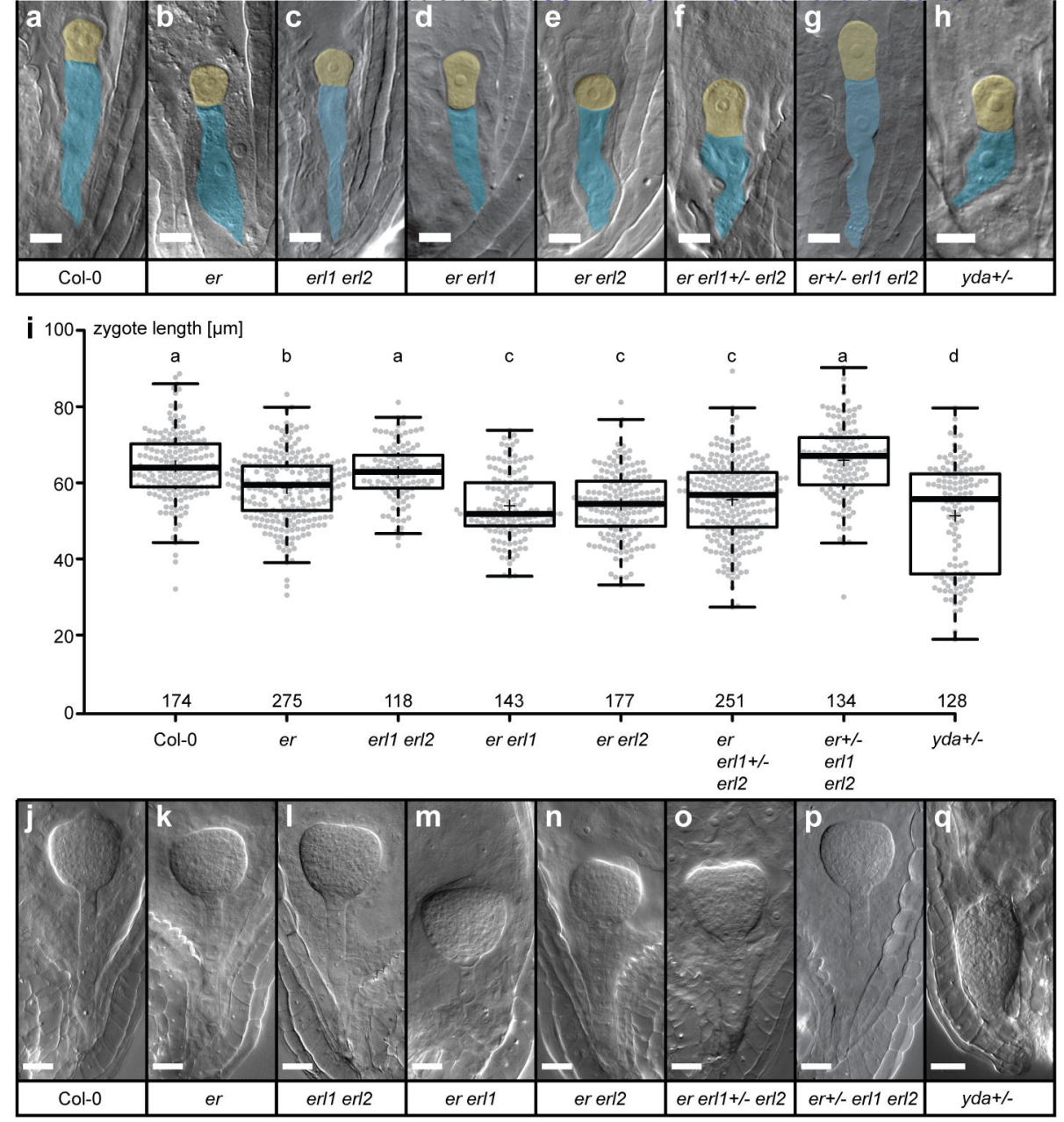

r suspensor length $[\mu \mathrm{m}]$

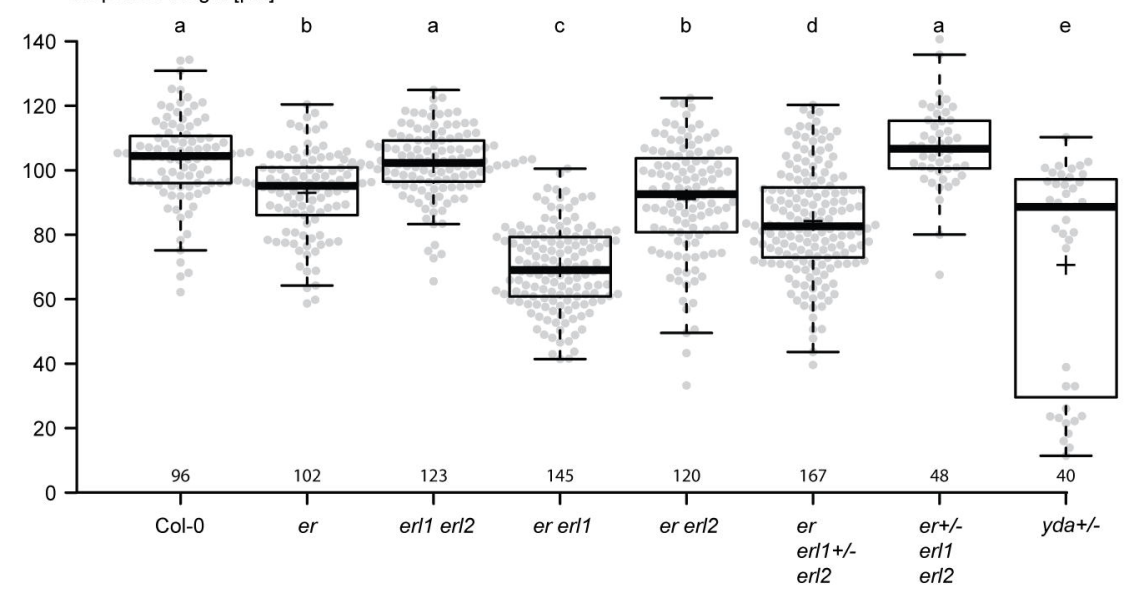

Figure 1: Zygote polarity and suspensor development

a-h) DIC images of cleared ovules showing representative 1-cell embryos. Apical cells are false colored in yellow, basal cells in blue. The genotype is given below the image. Heterozygous parental lines are indicated by $+/-$.

Scale bar $=10 \mu \mathrm{m}$.

i) Box plot diagram of zygote length in $\mu \mathrm{m}$. The sample size is given above $\mathrm{x}$-axis. Center lines show the medians; box limits indicate the 25th and 75th percentiles; whiskers extend 1.5 times the interquartile range from the 25 th and 75 th percentiles; crosses represent sample means; data points are plotted as grey dots. Statistical differences determined by Mann-Whitney U-test $(p<0.05)$ are indicated by different letters above graph. In embryos of heterozygous yda plants, two distinct populations of data points can be observed, with approximately a quarter showing strong zygote elongation defects possibly representing the homozygous offspring.

j-q) DIC images of cleared ovules showing transition stage embryos. The genotype is given below the image. Heterozygous parental lines are indicated by $+/$. Scale bar $=20 \mu \mathrm{m}$.

r) Box plot diagram of suspensor length at transition stage. The sample size is given above the x-axis. Center lines show the medians; box limits indicate the 25th and 75th percentiles; whiskers extend 1.5 times the interquartile range from the 25 th and 75 th percentiles; crosses represent sample means; data points are plotted as grey dots. Statistical differences determined by Mann-Whitney U-test $(\mathrm{p}<0.05)$ are indicated by different letters above graph. In embryos of heterozygous yda plants, two distinct populations of data points can be observed, with approx. a quarter showing reduced suspensor length possibly representing the homozygous offspring. 
a zygote length [ $\mu \mathrm{m}]$

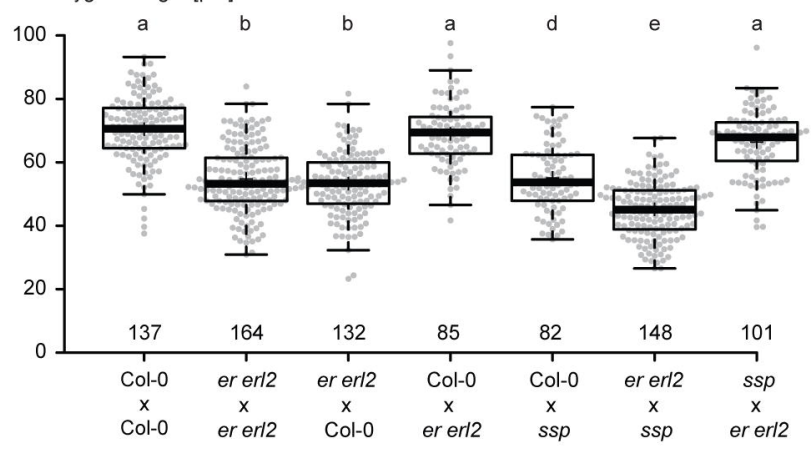

b suspensor length $[\mu \mathrm{m}]$

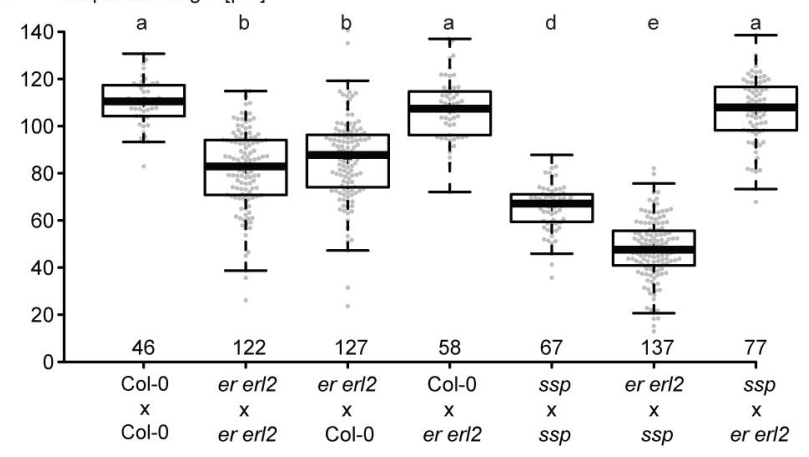

\section{Figure 2: Parental effects in reciprocal crosses}

Box plot diagrams of zygote elongation a) and suspensor length b) in $\mu \mathrm{m}$ in F1 embryos of reciprocal crosses.

The embryonic defects of the er erl 2 double mutant depends solely on the maternal genotype. The paternal effect of $s s p$ mutants enhances the er erl 2 defects in er erl 2 x ssp. The reciprocal ssp x er erl 2 cross does not show any detectable differences to wildtype.

Genotypes are given as female $x$ male. The sample size is indicated above the x-axis. Center lines show the medians; box limits indicate the 25th and 75th percentiles; whiskers extend 1.5 times the interquartile range from the 25th and 75th percentiles; small crosses represent sample means; data points are plotted as grey dots. Statistical differences determined by Mann-Whitney U-test $(p<0.05)$ are indicated by different letters above graph. 


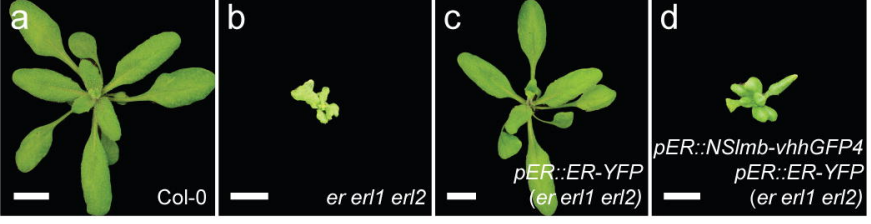

$\mathrm{e}$

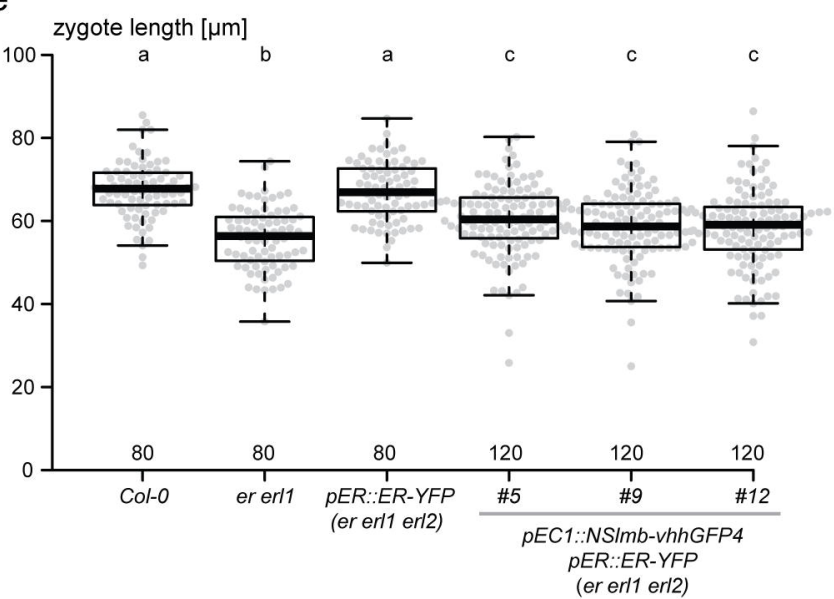

\section{Figure 3: Cell-autonomous function of $\mathbf{E R}$ protein in the zygote}

Vegetative growth phenotype of wild-type Col-0 (a), er erll erl2 triple homozygous (b), genetically rescued plants ( $p E R:: E R-Y P e t$ in er erl1 erl 2 ; c), and genetically recued plants expressing anti-GFP nanobody ( $E E R:: N S l m b-v h h G F P 4$ in $p E R:: E R-Y P e t$ er erl1 erl2; d). Scale bar $=1 \mathrm{~cm}$.

e) Egg cell-specific expression of the anti-GFP nanobody ( $p E C 1: \because N S l m b-v h h G F P 4)$ in the $p E R:: E R-Y P e t$ er erll erl2 plant leads to reduced zygote length. Box plot diagram of zygote elongation in $\mu \mathrm{m}$. The sample size is indicated above the $\mathrm{x}$-axis. Center lines show the medians; box limits indicate the 25th and 75th percentiles; whiskers extend 1.5 times the interquartile range from the 25th and 75th percentiles; small crosses represent sample means; data points are plotted as grey dots. Statistical differences determined by MannWhitney U-test $(\mathrm{p}<0.05)$ are indicated by different letters above graph. 

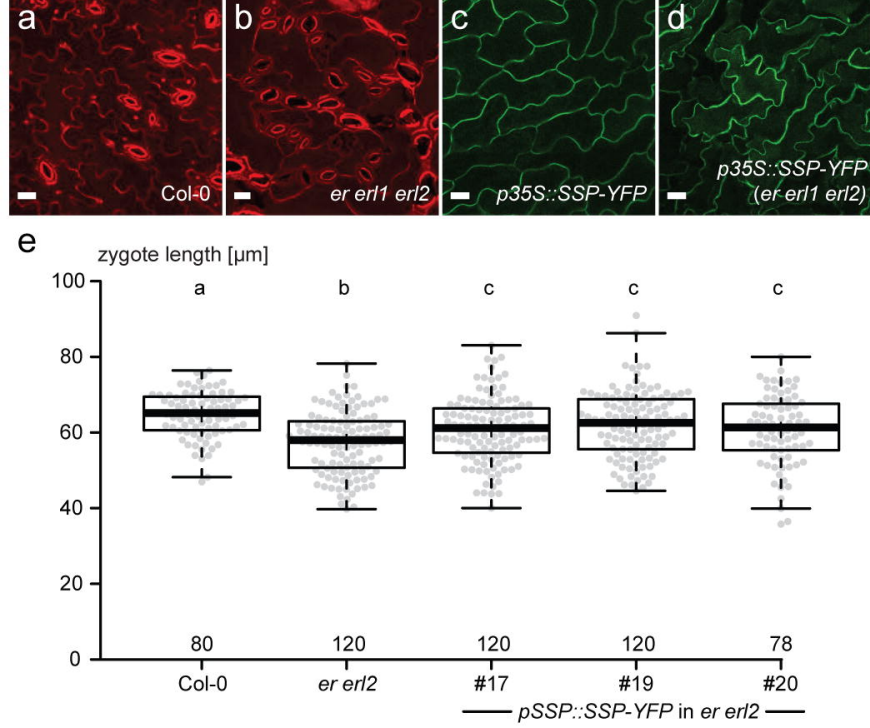

f

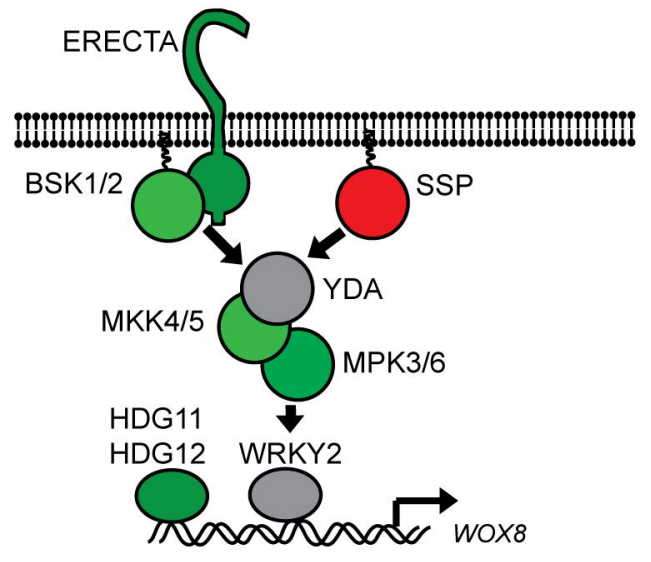

Figure 4: Y-shaped topology of the embryonic YDA pathway

Ectopic expression of SSP-YFP in leaves under control of the CaMV $35 S$ promoter ( $p 35 S:: S S P-Y F P$ ) leads to absence of stomata on the leaf surface in wild-type (c) and er erll erl2 background (d). Col-0 (a) and er erll erl2 (b) are shown as reference. SSP-YFP signal is shown in green, propidium iodide staining in red. Scale bar $=20 \mu \mathrm{m}$.

An additional copy of SSP ( $p S S P:: S S P-Y F P$ ) partially rescues the er erl2 loss-of-function phenotype in the zygote (e). Box plot diagram of zygote elongation in $\mu \mathrm{m}$. The sample size is indicated above the $\mathrm{x}$-axis. Center lines show the medians; box limits indicate the 25 th and 75 th percentiles; whiskers extend 1.5 times the interquartile range from the 25 th and 75 th percentiles; small crosses represent sample means; data points are plotted as grey dots. Statistical differences determined by Mann-Whitney U-test $(\mathrm{p}<0.05)$ are indicated by different letters above graph.

f) Simplified schematic depiction of the embryonic YDA pathway. YDA is activated by the maternally supplied receptor complex consisting of ERECTA and the membrane-associated proteins BSK1 and BSK2. YDA can also be activated by the paternally contributed BSK family member SSP. Signaling components are colored according to genetic contribution: maternal effect (green), paternal effect (red), zygotic recessive (grey). 